\title{
UN TROZO DE ITALIA EN EL CORAZÓN DE SINTRA: LA OBRA DE LUIGI MANINI PARA VICTOR CARLOS SASSETTI EN EL MONTE DA LUA
}

\author{
A PIECE OF ITALY IN THE HEART OF SINTRA: \\ THE WORK OF LUIGI MANINI FOR VICTOR CARLOS \\ SASSETTI AT MONTE DA LUA
}

Iván Moure Pazos

Universidade de Santiago de Compostela. España

ivan.menes@yahoo.es

\begin{abstract}
El presente artículo trata sobre la obra de Luigi Manini realizada para Victor Carlos Sassetti en la sierra de Sintra. El primer epígrafe se centra en el estudio pormenorizado de la Villa Sassetti, su papel como elemento generador de paisaje y su hipotética originalidad dentro del catálogo razonado de la obra del artista. El segundo apartado aborda la polémica surgida en torno a la autoría de los frescos realizados para el Hotel Victor de Sintra, supuestamente atribuidos a Manini, exponiendo, tras 42 meses de estudio en Italia y Portugal, nuestras conclusiones al respecto.

Palabras clave: Victor Carlos Sassetti; Villa Sassetti; Sintra; Luigi Manini; Hotel Victor.
\end{abstract}

This paper is about the works of Luigi Manini for Victor Carlos Sassetti at Sintra's mountain rage. The first epigraph focuses on the detailed study of Villa Sassetti, its role as a landscape generating element and its supposed originality within the author's reasoned catalog. The second section addresses the controversy about the authorship of the frescoes for the Hotel Victor at Sintra, allegedly attributed to Manini, exposing, after 42 months of study in Italy and Portugal, our conclusions.

Keywords: Victor Carlos Sassetti; Villa Sassetti; Sintra; Luigi Manini; Hotel Victor. 


\section{INTRODUCCIÓN}

Hace ahora exactamente 20 años -el 4 de diciembre de 1995- la UNESCO declaraba el Paisaje Cultural de Sintra Patrimonio Mundial de la Humanidad ${ }^{1}$. Entre las obras catalogadas que contribuyeron a tal distinción se encontraban varias de las aportaciones que el célebre escenógrafo y arquitecto italiano Luigi Manini (1848-1936) había legado a la sierra de Sintra, tanto como proyectista principal como en calidad de indispensable colaborador. Villa Sassetti (1890), Chalet Biester (1890) o la Quinta da Regaleira (1898) son, actualmente, parte firmante y fundamental del nutrido legado patrimonial sintrense ${ }^{2}$. En 1890, el afamado mecenas y empresario Victor Carlos Sassetti (1851-1915) encomienda a Manini el proyecto de su casa, ubicada a medio camino entre el espectacular Centro Histórico de Sintra y los célebres Palácio da Pena (1836) y Castelo dos Mouros (siglo VIII) ${ }^{3}$. Nacía así el gran proyecto de Villa Sassetti (Figura 1). Aunque modesta y sin mucho artificio -al modo de las grandes construcciones neolombardas-, se trataba, como veremos, de una obra nutrida de una enorme carga escenográfica y paisajística. Con motivo de su actual restauración exterior y reciente apertura de los jardines -a fecha de 26 de septiembre de 2015- creemos necesario un acercamiento intelectual que ponga de relevancia su enorme importancia artística como pieza clave e

${ }^{*}$ El presente artículo forma parte del proyecto internacional Laboratorio di Ricerca sulle Città (Istituto di Studi Superiori. Università di Bologna). Asimismo, también del proyecto europeo Performigrations: people are the territory (Dipartimento di Lingue, Letterature e Culture Moderne. Università di Bologna). De manera análoga, dicha ponencia ha sido posible gracias a la financiación postdoctoral del Plan Galego de Investigación, Innovación e Crecemento 2011-2015 promovido por la Xunta de Galicia. Toda la bibliografía del presente artículo se encuentra editada mayormente en italiano y portugués. Todos los textos citados han sido traducidos por el autor al castellano. La primera parte de este artículo correspondiente a la introducción y primer epígrafe -aunque actualizado en imágenes, texto y bibliografía-, ha sido presentado recientemente con el título de "Algunos apuntes sobre Villa Sassetti en Sintra" en el I Congreso Internacional de jóvenes investigadores. Coleccionismo, mecenazgo y mercado artístico en España e Iberoamérica celebrado el 15-18 de febrero en la Facultad de Geografía e Historia de la Universidad de Sevilla. Lo que ahora se ofrece es una versión ampliada de esta primera investigación, cuyo fin totalizador se centra en el análisis pormenorizado de la obra de Manini realizada para el célebre empresario y mecenas Victor Carlos Sassetti en la ciudad de Sintra.

${ }^{1}$ VON DROSTE, Bernard et al.: Organisation des nations unies pour l'education, la science et la culture. Convention concernant la protection du patrimoine mondial, culturel el naturel. Comite du patrimoine mondial. Dix-neuvième session. Berlin, UNESCO, 4-5 décembre 1995, p. 46.

2 AA.VV.: Sintra, Património da Humanidade. Sintra, 1996, p. 13.

${ }^{3}$ PEREIRA, Denise y LUCKHURST, Gerald: "A Vila Sassetti, Projecto do cenógrafo-arquitecto Luigi Manini", Varia Escrita, 11, p. 258.

${ }^{4}$ LUCKHURST, Gerald y CORNÉLIO DA SILVA, José: Sintra, A Paisagem e suas Quintas. Lisboa, 1989, p. 114. 
indispensable del profuso recetario sintrense contemporáneo. Análogamente y, a pocos metros de esta gran Villa, nos encontramos, actualmente, con varias obras supuestamente atribuidas a Manini -todavía sin fechar- realizadas para el antiguo Hotel Victor de Sintra - propiedad empresarial de Victor Carlos Sassetti-. Cabe apuntar que no existe bibliografía específica sobre estas obras que, a nuestro juicio, merecen al menos una primera mención articulística. Este caso concreto será tratado pormenorizadamente en un epígrafe subsiguiente, a fin de arrojar luz sobre un tema tan polémico como apasionante que vendría a poner en solfa, una vez más, la estrecha relación existente entre Victor Carlos Sassetti y Luigi Manini en la ciudad de Sintra; dos italianos encontrados en aquella eterna fiesta de fastos que era, por entonces, el fin de siècle sintrense.

\section{CUANDO LA ARQUITECTURA DEVIENE EN ESCENOGRAFÍA Y UNA ACUARELA INESPERADA}

La Villa Sassetti, hoy catalogada por la UNESCO como "Valor Fundamental" de la arquitectura áulica sintrense, constituye, en palabras de los especialistas Denise Pereira y Gerald Luckhurst, "el proyecto más escenográfico de la carrera de Luigi Manini"'s. Sobre ello incidíamos en un artículo de reciente aparición pública titulado "Las Villas de Luigi Manini en Sintra (1890-1912)":

"Redundar en que la arquitectura de Manini resulta altamente escenográfica se antoja una evidencia incuestionable. La Villa Sassetti constituye un ejemplo claro de este juego de perspectivas teatrales. Desde cualquiera de los grandes monumentos sintrenses - exceptuando el Palácio de Monserrate (1858)-: Paço da Vila (1489), Castelo dos Mouros (siglo VIII), Chalet Biester (1891), Palácio da Pena (1836) y Quinta da Regaleira (1898-1912), se divisa, imponente, la Villa Sassetti. Pudiéramos atribuir este hecho al azar, pero lo cierto es que resulta poco factible -a tenor de las distancias existentes entre los monumentos y la escasa visibilidad que ofrece la frondosidad vegetal de la Sierra- que tal alineamiento sea una coincidencia; máxime sabiendo que en las restantes creaciones de Manini esto se revela como un leitmotiv recurrente. De tal modo que se establece una correlación escenográfica entre los principales monumentos, a través de un interesante juego de perspectivas; de un verdadero genius loci global, inmortalizado en las fotografías que el autor, en sus frecuentes paseos por la sierra, nos ha legado"6.

Podríamos ir más allá y afirmar que desde ese gran mirador que son los jardines de la edénica Villa Sassetti, también se avizoran la antiquísima Quinta dos Pisões (1533), las dieciochescas Quinta de São Sebastião (1780) y Palácio de

\footnotetext{
${ }^{5}$ PEREIRA, D. y LUCKHURST, G.: "A Vila Sassetti...”, op. cit., p. 270.

${ }^{6}$ MOURE PAZOS, Iván: "Las Villas de Luigi Manini en Sintra (1890-1912)”, Ángulo Recto. Revista de estudios sobre la ciudad como espacio plural, vol. 6, nº 2, p. 107.
} 
Seteais (1787), así como las "románticas" Quinta do Saldanha (1830), Quinta do Relógio (1850) o Villa Roma (años centrales del siglo XIX), entre otras muchas obras de incalculable valor artístico que hacen de Sintra un auténtico museo palacial (Figuras 2-3). Todo ello, pese a que "el crecimiento acelerado de la vegetación envolvente y, en particular, la adición del muro de poniente suprimieran las perspectivas visuales que existían al nivel del piso terreno"7, como bien podemos apreciar en algunas pinturas de época (Figura 4). A esto nos referíamos cuando afirmábamos que Manini había sometido "la arquitectura a extremos de una irresistible visualidad, a través de una premeditada y cuidada búsqueda de la potencialidad del lugar" ${ }^{8}$. Desde un punto de vista artístico, el proyecto de Villa Sassetti debe su éxito a esta gran premisa teatral y escenográfica, actuando como indudable elemento generador de paisaje. Pudiera pensarse también, como es lógico, que en el contexto orográfico de una sierra, cualquier obra situada a mayor altura de las restantes ofrece una vista privilegiada de las mismas, pero lo cierto es que Villa Sassetti establece esta misma correspondencia axial con sus superiores Castelo dos Mouros y Palácio da Pena. Esto no es baladí, pues, el eje que enlaza Sassetti con la Pena se antoja extremadamente rebuscado, alejándonos de cualquier tesis casual. Antes bien, debiéramos "abrazar" la hipótesis premeditada y causal -de clara intencionalidad antrópica- basada en que Manini, "emulando a los grandes artistas renacentistas [...] dispuso de una ciudad para convertirla y predefinirla en arte, imprimiendo en ella su ideal de belleza así como su concep-

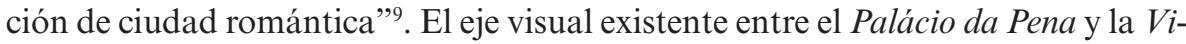
lla Sassetti debe de ser registrado como un auténtico logro paisajístico. Estamos ante un ángulo imposible sobre el cual debiéramos reflexionar ${ }^{10}$. Villa Sassetti se levanta sobre un terreno de extrema verticalidad que se incrementa a medida que se gana en altura. La cumbre, coronada por el Castelo dos Mouros y sus penedos subsecuentes, se levanta "sobrevolando" Sassetti en una suerte de perspectiva casi aérea. Se trata de una perpendicularidad vertiginosa. La imponente presencia de los Mouros se erige como una gran pantalla mural, agotando la perspectiva visual de Sassetti en dirección al otro gran promontorio sintrense entronizado por el majestuoso Palácio da Pena. Pero la genialidad de Manini, cuando se trata de idear paisajes, no tiene parangón. El artista cremonese erige la torre principal de Sassetti en la vertiente oeste de la ladera de los Mouros, salvando -por cuestión de metros- el gran obstáculo del Penedo da Amizade; un enorme pedernal de más de 45 metros de altura, utilizado de enmarque lateral para el encuadre de la perspectiva de la Pena. Esto permite la creación de un recuadre -casi fotográfico-de

${ }^{7}$ PEREIRA, D. y LUCKHURST, G.: “A Vila Sassetti...”, op. cit., p. 262.

${ }^{8}$ MOURE PAZOS, I.: "Las Villas de Luigi Manini...", op. cit., pp. 114-115.

9 Ibidem, p. 115.

${ }_{10}$ Actualmente entorpecido por la gigantesca y exuberante frondosidad vegetal de la sierra. 
dicho Palácio, tomado desde la torre principal de Sassetti. Se trata de una recurso teatral único, bien medido y perfectamente estudiado que "agujerea" la sierra sintrense de lado a lado a lo largo de aproximadamente un kilómetro de longitud en línea recta, salvando un desnivel de varios cientos de metros de altura. Al caer la noche, recortado en luz sobre un fondo negro, las vistas de la Pena desde Sassetti resultan bellísimas a la par que espectrales. Se trata de un espectáculo de alta escenografía que "apaga" el paisaje y "enciende" la arquitectura, enfatizando, más si cabe, el rebuscado eje visual entre sendas obras.

Con acierto, Denise Pereira y Gerald Luckhurst han considerado el enclave de Sassetti como "un lugar aislado y solitario, casi inaccesible, de rusticidad bucólica y pintoresca, abierto a la contemplación exógena y endógena del hombre y del paisaje" ". Ya hemos apreciado cómo la torre circular de la Villa-horadada por innúmeros vanos en su parte alta-, se erige como un auténtico mirador sintrense, ofreciéndonos una de las mejores recreaciones panorámicas de la sierra y su centro histórico. Quizás, mejores panorámicas, desde un punto de vista patrimonial, que las ofrecidas por el Palácio da Pena, obra señera de indudable superioridad artística, pero privada de las maravillosas vistas a la Vila Velha -el corazón de Sintra- con su elenco de palacios aristocráticos y las más ingeniosas quintas burguesas. Por lo tanto, es aquí donde reside la fuerza de la obra que nos ocupa. Sassetti actúa como un enorme catalizador visual, "animando" las líneas de paisaje que cruzan la sierra sintrense. E incidíamos en este aspecto porque en lo arquitectónico, Sassetti carece de una gran complejidad artística. Se trata de una "modesta" obra boitiana realizada -en palabras del propio Manini- en stile lombardo ${ }^{12}$ :

"Boito, que impartió clases en la Accademia di Brera -donde Manini estudió-, realiza en esta época una apología del estilo lombardo-bizantino definiéndolo como el estilo más representativo de la tradición italiana, incitando al estudio compulsivo de los monumentos de arquitectura románica [...] eligiendo el estilo lombardo de la Alta Edad Media como un estilo rico, severo y exclusivamente italiano. En un momento en que el lenguaje arquitectónico se convertía en un instrumento de apelación y valorización del débil sentimiento de identidad nacional, el stile lombardesco o comacino se reveló como una arquitectura genuina carente de elementos foráneos [...]"13.

Al modo de un gran maestro comacino, Manini, erige un monumento neolombardo en el corazón de Portugal. Por entonces, este país contaba con su propio estilo "preservacionista" exaltador del ánima lusitana, el llamado nenomanuelino, del cual, andando el tiempo, Manini se convertirá en su mayor exponente

${ }^{11}$ PEREIRA, Denise, LUCKHURST, Gerald: "Luigi Manini e o projecto da Vila Sassetti em Sintra", Monumentos, vol. 26, p. 245.

${ }^{12}$ PEREIRA, D. y LUCKHURST, G.: "A Vila Sassetti...”, op. cit., p. 264.

13 Ibidem, p. 239. 
a través de las grandiosas aportaciones para Buçaco (1888-1907) y la Quinta da Regaleira (1898-1912) ${ }^{14}$. Pese a lo que pudiera parecer, la obra no carecía de sentido, pues no debemos olvidar que la iniciativa del mecenas Victor Carlos Sassetti era en origen italiana, al igual que su hacedor, como sabemos, natural de Lombardía. Como telón de fondo de este revival y, amén de Boito, Denise Pereira y Gerald Luckhurst, han considerado tres libros fundamentales que pudieran haber influenciado a Manini en esta empresa neolombarda ${ }^{15}$. Se trata de Étude sur l'architecture lombarde et sur les origines de l'architecture romano-byzantine (1865) y Architecture lombarde par F. de Dartein (1892), ambos firmados por Fedinand de Dartein, así como Elementi di architettura lombarda redatti da Edoardo Mella (1885) bajo rúbrica de Edoardo Arborio Mella ${ }^{16}$. Dicha tratadística incidía mayormente, y como es lógico, en el estudio de las grandes producciones medievales

${ }^{14}$ MOURE PAZOS, I.: “Las Villas de Luigi Manini...”, op. cit., pp. 111-114.

15 PEREIRA, D. y LUCKHURST, G.: "A Vila Sassetti...”, op. cit., p. 238.

${ }^{16}$ DE DARTEIN, Fedinand: Étude sur l'architecture lombarde et sur les origines de l'architecture romano-byzantine. Paris, 1865; DE DARTEIN, Fedinand: Architecture lombarde par F. de Dartein. Paris, 1892; y MELLA, Edoardo Arborio: Elementi di architettura lombarda redatti da Edoardo Mella. Torino, 1885. Debemos añadir a estas referencias un compendio de libros fundamentales, estrechamente vinculados a todo el conjunto de la obra maniniana: ALBERTOLLI, Giocondo: Ornamenti diversi, inventati disegnati ed eseguiti da Giocondo Albertolli: Professore d'Ornati nella Reale Accademia di Belle Arti in Milano. Incisi da Giacomo Mercoli Luganese. Milano, 1782; ALBERTOLLI, Giocondo: Alcune decorazioni di nobili sale ed altri ornamenti di Giocondo Albertolli: Professore nella Reale Accademia delle Belle Arti in Milano. Incisi da Giacomo Mercoli e da Andrea de Barnardis. Milano, 1787; ALBERTOLLI, Giocondo: Miscellanea per i giovani studiosi del disegno pubblicata da Giocondo Albertolli: Professore nella Reale Accademia delle Belle Arti in Milano. Milano, 1796; ALBERTOLLI, Giocondo: Corso elementare di ornamenti architettonici ad uso de'principianti. Milano, 1805; MOGLIA, Domenico: Collezione di soggetti ornamentali ed architettonici inventati e disegnati da Domenico Moglia. Milano, 1837; VIOLLET-LE-DUC, Eugène: Dictionnaire raisonné de l'architecture française du XIe au XVIe siècle (1854-1868). París, 1868; VIOLLET-LE-DUC, Eugène: Dictionnaire raisonné di mobilier française de l'epoque carlovingienne à la Renaissance. París, 1874; BOITO, Camilo: Architettura del Medio Evo in Italia, con una introduzione sullo stile futuro dell'architettura italiana. Milano, 1880; SACCHI, Archimede: Le abitazioni: alberghi, case operaie, fabbriche rurali, case civili, palazzi e ville: ricordi compendiati da Archimede Sacchi. Milano, 1874; y CASSINA, Ferdinando: Le fabbriche più cospicue di Milano, pubblicate per cura di Ferdinando Cassina. Milano, 1840. Para todo aquel interesado en la relación de Luigi Manini con esta tratadística se recomienda la consulta de las siguientes obras: MOURE PAZOS, Iván: "Primeros apuntes sobre la Quinta da Regaleira de Luigi Manini: el Palacio (1893-1912)", Arbor (en prensa); MOURE PAZOS, Iván: "El Mausoleo de los Carvalho Monteiro de Luigi Manini en el Cemitério dos Prazeres de Lisboa", en Actas de las VII Jornadas Arte y Ciudad (IV Encuentros Internacionales) (en prensa); y MOURE PAZOS, Iván: "Manini diseñador en la Quinta da Regaleira: el lenguaje clásico de una obra neomanuelina (1898-1912)", Arte y Ciudad (en proceso de revisión por pares ciegos). 
de origen religioso. Ahora Manini, con gran maestría, transformaba estas "conquistas" lombardas, en pura arquitectura finisecular burguesa ${ }^{17}$. Pero lo cierto es que al margen del estudio tratadístico, Manini llevaba ensayando desde el inicio de su carrera algunos dibujos y pinturas que en mucho se asemejaban a su proyecto para Sassetti. Debemos apostillar que entre los géneros pictóricos preferidos por Manini se contaba la pintura de paisaje, preferentemente de temática montañosa y alpina y, a ser posible, con un castillo medieval coronando la escena. Este tema se convertirá, a lo largo de su dilatada carrera, en una de las señas identitarias de toda su producción artística. El paisaje publicado en el número 135 de la revista Occidente $(1882)^{18}$, sus trabajos escenográficos para el Tannhäuser (1891-1892) de Richard Wagner (1813-1883) ${ }^{19}$, o la magnífica tela titulada Paesaggio ideale con castello (post 1910-ante 1936) ${ }^{20}$, entre muchas otras, son sintomáticas de esta preocupación por la arquitectura medieval y los paisajes evocadores de sabor puramente pintoresquista.

Mención aparte merece una extraña acuarela de Manini fechada en torno a 1862 intitulada Veduta fantastica di città con castello o bien Paesaggio lacustre con architetture, donada en 1998 por Elda Manini al Museo Civico di Crema e del Cremasco. Se trata de una obra de enorme importancia para el estudio de Manini, pues, estamos ante una reproducción casi clónica de la obra que nos ocupa. Baste una simple comparativa visual para advertir evidentes similitudes entre sendas obras (Figuras 5-6). Bandas lombardas, arcos ciegos, mampostería, ajimeces, chimeneas, cubiertas, anexos torreados y hasta la torre circular en resalte a modo de pequeño donjon con su característica sucesión de vanos en su parte alta, nos transportan, indefectiblemente, a Villa Sassetti. Esto convierte este discreto y desconocido documento acuarelado en una pequeña joya maniniana de enorme valía artística, afianzando, más si cabe, la querencia del cremonese por el stile lombardesco de su añorada tierra natal y sus evocadores paisajes. Estamos ante el gusto pintoresco tan característico de su obra, consustancial, como sabemos, a toda su producción pictórica, arquitectónica y escenográfica.

17 Aspecto presente también en el segundo proyecto frustrado presentado para su mecenas en 1894. Vid. PEREIRA, D. y LUCKHURST, G.: "Luigi Manini e o projecto...", op. cit., pp. 243-244.

${ }_{18}$ MANINI, Luigi: "Desenho", O occidente: revista illustrada de Portugal e do estrangeiro, vol. 5, n 135, p. 1. Vid. PEREIRA, D. y LUCKHURST, G.: "A Vila Sassetti...", op. cit., p. 264.

19 MOURE PAZOS, Iván: "Primeros apuntes...”, op. cit.

${ }^{20}$ ALPINI, Cesare: "Manini Pittore", en Gaia Piccarolo y Giuliana Ricci (coords.): Luigi Manini (1848-1936) architetto e scenografo pittore e fotografo. Milán, 2007, p. 125. Alpini nos aclara que dicha obra fue donada por el doctor Gallini al museo de Crema donde se encuentra actualmente. 


\section{POSIBLES FRESCOS DE MANINI PARA EL HOTEL VICTOR DE SINTRA}

A escasos metros de la entrada principal de la Villa Sassetti nos encontramos con el antiguo Hotel Victor; uno de los hoteles más lujosos de la época, propiedad, en su momento, de Victor Carlos Sassetti ${ }^{21}$, "por donde pasaron, a finales del siglo XIX, Eça de Queiróz, Ramalho Ortigão y donde Alberto Pimentel escribió su libro Noites de Sintra. El hotel sintrense, descrito en las páginas queirosianas como un lugar elegantísimo, fue frecuentado por la más alta sociedad del momento, cuyas estadías merecían destaque en los jornales regionales. Estuvo en funcionamiento desde la década de 1850 hasta 1890, altura en que las estancias de veraneo en las costas de Cascais y de los Estoris se impusieron como principales destinos de moda, finalizando así la primacía sintrense como lugar predilecto de descanso estival"'22.

En el mismo Hotel Victor se encuentran una serie de frescos atribuibles a Manini que, todavía hoy, continúan siendo motivo de controversia y especulación. Actualmente -admitámoslo- no podemos fechar con exactitud el año de realización de la obra, siendo también imposible identificar con seguridad al autor o autores de la misma. Por lo tanto, debemos ser muy cautos y trabajar siempre desde el terreno de la suposición y la conjetura. La falta total de producción científica al respecto tampoco ayuda. Sin embargo, tras varios años rastreando la versátil figura de Manini en Italia y Portugal, debemos afirmar que dicha obra pudiera ser fácilmente atribuible al artista cremonese. Tal afirmación debe de ser humilde y detalladamente argumentada. Pero antes de comenzar, quisiéramos hacer un inciso y mencionar que esperamos con entusiasmo e impaciencia la publicación de las primeras investigaciones, todavía en curso e inéditas, de la profesora Ana María da Silva Lourenço Paiva, persona vinculada laboralmente al antiguo Hotel Victor y gran conocedora de la realidad sintrense del XIX, cuyas hipótesis se inclinan también a favor de una autoría maniniana en base a argumentos valiosísimos que, esperamos, vean la luz pronto. En espera de nuevos datos científicos que ayuden al esclarecimiento definitivo de esta polémica historiográfica y, deseando la pronta aparición pública de estas investigaciones, expondremos a continuación una primera síntesis de nuestro estudio.

Los frescos atribuidos a Manini se distribuyen en diversos paneles, alternando diferentes escenas paisajísticas de la ciudad de Sintra con algunas representaciones alpinas y prealpinas de su Lombardía natal. Como hemos comentado con anterioridad, resulta difícil, si no imposible, datar con precisión el año de realización de estos frescos, sin embargo, creemos que éstos fueron elaborados durante

${ }^{21}$ He de agradecer encarecidamente al escritor y dinamizador cultural sintrense don Miguel Boim, el haberme revelado la existencia de estas obras durante mi estancia de investigación postdoctoral en tierras lusas.

${ }^{22}$ PEREIRA, D. y LUCKHURST, G.: "A Vila Sassetti...”, op. cit., p. 256. 
la década de 1880. El Hotel Victor estuvo en activo entre 1850 y 1890, y sabemos con certeza que Manini recala por primera vez en Portugal en el año 1879, cuando es requerido como afamado escenógrafo en el Real Teatro San Carlos de Lisboa $^{23}$. Recién llegado a la capital lusa, Manini establece una rápida y estrechísima relación con Victor Sassetti ${ }^{24}$. No olvidemos que ambos eran de origen italiano -aunque cabe apuntar que este último había nacido ya en Sintra-. Por aquel entonces, Sassetti regentaba uno de los hoteles más refinados de Lisboa, el Hotel Braganza -en cierto modo, homólogo en lujo y opulencia al Hotel Victor de Sintra que nos ocupa-, "frecuentado por ilustres huéspedes de la aristocracia portuguesa, de la vida política, diplomática y de la ópera, el Braganza, fue inmortalizado por algunos de los mayores representantes de las letras nacionales. En él se hospedaron muchos jefes de estado, D. Pedro II de Brasil, la Reina de Suecia, el Sultán de Zanzíbar, y la diva escénica Sarah Bernardt que se alojaría en el Braganza durante largas temporadas" 25 .

Es muy probable que la amistad entre ambos se cimentara en torno al efervescente ambiente operístico, marcadamente elitista, de la alta sociedad lisboeta del momento. Tal fue así que en 1888, Sassetti encomienda a Manini la realización de diversas pinturas para su Hotel Braganza ${ }^{26}$. No sería disparatado, por lo tanto, presuponer que el célebre mecenas y empresario de origen italiano hiciese lo propio con su homólogo Hotel Victor de Sintra, años antes de encomendarle al artista la realización de Villa Sassetti. Debemos recordar que Victor Sassetti fue, conjuntamente a António Augusto Carvalho Monteiro (1848-1920) y Frederico Biester (1833-1899), uno de los principales mecenas del artista cremonese en Portugal; país en el que Manini trabajará sin descanso durante más de 30 años $^{27}$. Por consiguiente, y a juzgar por la enorme admiración que Sassetti profesaba a

${ }^{23}$ PEREIRA, Denise y LUCKHURST, Gerald: "Cenografia e espectáculo", en CRUZ ALVES, Joao (coord.): Luigi Manini: Imaginário y Método, Arquitectura y Cenografia. Sintra, 2006, p. 44.

${ }^{24}$ La relación entre Manini y Sassetti debe considerarse más allá de lo estrictamente profesional. Ésta fructificará en una estrecha amistad que perdurará hasta la muerte del empresario acaecida abruptamente en el año 1915. Existen testimonios gráficos de las visitas de los Manini en casa de los Sassetti. También debe apuntarse que, cuando el artista cremonese inicie en Sintra su obra más ambiciosa, la Quinta da Regaleira, se alojará en una de las casas de la sierra propiedad del empresario. Tampoco debemos pasar por alto que en 1902, Ebe Manini, hija del artista, y su marido Azelio Bacchetta pasarán su luna de miel en la Villa Sassetti. Vid. PEREIRA, D. y LUCKHURST, G.: "Biografía”, op. cit., pp. 269-270.

${ }^{25}$ PEREIRA, D. y LUCKHURST, G.: "A Vila Sassetti...”, op. cit., p. 254.

${ }^{26}$ PEREIRA, D. y LUCKHURST, G.: "Biografía”, op. cit., p. 269; y PEREIRA, D. y LUCKHURST, G.: "Luigi Manini: esboço de uma biografia”, op. cit., p. 14.

${ }^{27}$ MOURE PAZOS, Iván: "El programa iconográfico de Manini y Monteiro para los jardines de la Quinta da Regaleira (Parte I)”, Ars Longa (en prensa); MOURE PAZOS, I.: 
Manini, es de toda lógica presuponer su firma en dichos frescos, siendo éstos realizados durante la horquilla cronológica anteriormente mencionada; concretamente, creemos que 2 o 3 años antes de la realización de sus magníficas pinturas decorativas para el edificio principal del Chalet Biester-vasto complejo en el que Manini proyectará también la Casa del Giardiniere-Biester (1890-1891) $)^{28}$ y sus aportaciones artísticas para el Hotel Braganza ${ }^{29}$. A este respecto, no podemos pasar por alto un dato de suma relevancia que vendría a secundar y reforzar nuestra hipótesis cronológica. Al analizar los frescos del Hotel Victor, advertiremos que las escenas paisajísticas referidas a Sintra carecen de representaciones arquitectónicas posteriores a 1890, es decir, las vistas se componen solamente de obras anteriores a esta fecha, tales como el Castelo dos Mouros (siglo VIII), el Palacio da Pena (1836), el Palacio de Monserrate (1858) o la Quinta do Relógio (1850), omitiéndose, en todo caso, clásicos de la sierra sintrense como la Villa Sassetti (1890), la Quinta da Regaleira (1898-1912) o el mismísimo Chalet Biester (1890). Todo ello, quizás, debido a que durante el año de realización de la obra, dichos edificios todavía no habían sido proyectados, permitiéndonos establecer una fecha límite y aproximativa en la ejecución de las pinturas.

Pero hasta el momento, y a falta de pruebas estrictamente científicas, la aportación más fidedigna a la hora de valorar dicha atribución, la encontraremos en el estudio formal y detallado de la obra que nos ocupa. Al final del epígrafe precedente, subrayábamos la querencia de Manini por las panorámicas fragosas y montañosas, tanto en su faceta de pintor como de reputado escenógrafo y arquitecto. En la sección de frescos dedicados a los paisajes lacustres prealpinos del Hotel Victor -conjunto que, sin duda, debió agradar sobremanera a su supuesto mecenas- Manini parece delatarse. La similitud de estos frescos con la serie de pinturas realizadas para la Villa Stramezzi (principios del siglo XVI) en Moscazzano (Cremona), firmadas por Manini en 1867, resulta incuestionable ${ }^{30}$. Del mismo modo que gran parte de los Paesaggi de su primera etapa italiana pudieran vincularse holgadamente a la obra objeto de estudio. Paesaggio montano con muche, Paesaggio alpino con laghetto, o los dos Paesaggi pertenecientes a la Banca Popolare di Crema ${ }^{31}$, son ilustrativos de esta nutrida tradición paisajera, que se inicia en Villa Stramezzi, pasa por los posibles frescos del Hotel Victor, y se cierra con Paesaggio del Lago d'Iseo (1930), obra de madurez realizada por Manini a los 82 años de edad durante su etapa de retiro bresciano (Figuras 7-10). $\mathrm{Y}$ es que, baste una breve comparativa visual para advertir evidentes

"El Mausoleo de los Carvalho Monteiro...", op. cit.; y MOURE PAZOS, I.: "Manini diseñador en la Quinta da Regaleira...", op. cit.

${ }^{28}$ MOURE PAZOS, I.: "Las Villas de Luigi Manini...”, op. cit., pp. 103-106.

${ }^{29}$ PEREIRA, D. y LUCKHURST, G.: "Biografía", op. cit., p. 269.

${ }^{30}$ ALPINI, C.: "Manini Pittore", op. cit., p. 121.

31 Ibidem, p. 122. 
concomitancias formales entre su prolífico catálogo de obra prealpina y las supuestas pinturas para el Hotel Victor, es decir; composición, color, pigmentos, trazos, modelado, y luz, obran de igual manera en las distintas producciones, estableciéndose una clara homogeneidad totalizadora en el conjunto final. Se trata de correspondencias formales altamente sospechosas en relación a su producción paisajística anterior y posterior. Pero todavía resulta más inequívoco el hecho de que los paisajes del Hotel Victor se inspiren en localizaciones prealpinas lombardas estrechamente vinculadas al patrimonio afectivo maniniano. Se trata de lugares próximos a las diferentes residencias de los Manini, donde estos habitaron durante más de 40 años. Manini acostumbraba a "salpimentar" sus paisajes dal vero con algún motivo de corte pintoresquista que enfatizaba, más si cabe, el carácter escenográfico característico de su obra. Sin embargo, los marcos espaciales de las pinturas para el Hotel Victor resultan fácilmente reconocibles a los ojos de cualquier persona familiarizada con los generosos paisajes lacustres de la región lombarda. Concretamente hablamos de localizaciones muy precisas que, creemos, consciente o inconscientemente, pudieran estar representadas en los frescos del Hotel Victor. Se trata, posiblemente, de la pequeña localidad de Bellagio en Lago di Como; la zona medianera del Lago d'Iseo, referida a sus intersecciones con las tres islas centrales de Monte Isola, Isola di San Paolo e Isola di Loreto; y -menos probable aunque no descartable- una de las localizaciones menos conocidas del bellísimo Lago Maggiore en su extremo norte y piamontés, los llamados Castelli di Cannero.

\section{CONCLUSIONES}

Abríamos la presente investigación reflexionando sobre la enorme importancia de la obra maniniana en la sierra de Sintra, algo que, como sabemos, contribuyó a su nombramiento como Patrimonio Mundial de la Humanidad (UNESCO). Entre los "Valores Fundamentales" que posibilitaron tal mención se encontraba Villa Sassetti. Una obra que puso en solfa la gran potencialidad escenográfica de la arquitectura maniniana. El alineamiento con gran parte de los monumentos precedentes de la sierra, nos sitúa ante una obra maestra del paisaje contemporáneo. En Villa Sassetti, la búsqueda de un adecuado genius loci se erige como el aspecto fundamental y definitorio de la obra, alcanzando panorámicas y perspectivas del todo excepcionales. El remarque y la puesta en valor del Castelo dos Mouros, Paço da Vila, Quinta dos Pisões, Chalet Biester, Quinta de São Sebastião, Quinta do Saldanha, Quinta do Relógio, Villa Roma, Palácio da Pena o Palácio de Seteais, entre otros, son sintomáticos del gusto meticuloso de Manini por una arquitectura supeditada al paisaje. Entre todas estas recreaciones panorámicas, debemos de resaltar una en particular que, a nuestro modo de entender, raya la genialidad. Se trata de ese gran eje visual que engarza con el Palácio da Pena, bordeando, en un juego casi ilusionista, el espectacular Penedo da Amizade. Al 
margen de la tratadística neomedieval de Ferdinand de Dartein y Edoardo Mella -que el artista estudia con ahínco-, la Villa Sassetti permite a Manini la materialización tridimensional de algunos ensayos pictóricos previos. El paisaje para la revista de Occidente, o el Tannhäuser de Wagner entre otras pinturas y dibujos del género, acusa la evidencia de esta continuidad temática. Especial mención merece la magnífica acuarela Veduta fantastica di città con castello, por ser ésta una representación casi clónica del proyecto para Villa Sassetti. A escasos metros del gran pedernal, en el Hotel Victor, Manini -probablemente- realiza para Sassetti una serie de frescos de temática sintrense y prealpina. La cronología del hotel, contrastada con la documentación biográfica de Manini, y la omisión de ciertas obras sintrenses en la representación de los paneles, cercan la horquilla cronológica en torno a la década de 1880. La estrecha amistad del artista con Victor Sassetti, así como el encargo de las pinturas para el homólogo Hotel Braganza de Lisboa en 1888, orientan también hacia una autoría maniniana. Sin embargo, la mayor evidencia de dicha autoría, la encontramos en la serie lacustre de paisajes prealpinos. Diferentes aspectos de tipo formal, contrastados con obras anteriores y posteriores del profuso catálogo razonado de Manini, balizan el sendero de nuestras hipótesis. Sus trabajos para la Villa Stramezzi, así como el innúmero recetario de paesaggi que Manini desarrollla a lo largo de toda su etapa artística, nos sitúan, de manera clara, sobre la pista del artista cremonese. Las diferentes localizaciones lacustres y prealpinas de su Lombardia natal se antojan demasiado coincidentes para no ser consideradas una auténtica certeza; un dato sumamente sospechoso, que invita u obliga al inmediato análisis científico de las pinturas objeto de estudio.

Entre 1885 y 1890, Manini y Sassetti llenaron Sintra de instantáneas italianas, inicialmente con algunas panorámicas inspiradas en los Lagos de Como, d'Iseo, y Maggiore, posteriormente, erigiendo una villa neolombarda en el corazón de la sierra. Tan solo ocho años después, António Augusto Carvalho Monteiro encomendaría a Manini la realización de su obra más ambiciosa en la sierra sintrense, la Quinta da Regaleira, que convertirá al artista en uno de los arquitectos más solícitos y admirados de todo el Portugal fin de siècle, reportándole, andando el tiempo, una más que merecida fama internacional. Lejos quedarían aquellas primeras aportaciones sentimentales e italianas, mucho más humildes que, de la mano de Victor Carlos Sassetti, Manini erigió en la Villa de Sintra a finales de la década de 1880 .

Fecha de recepción: 30 de marzo de 2016

Fecha de aceptación: 31 de enero de 2017 


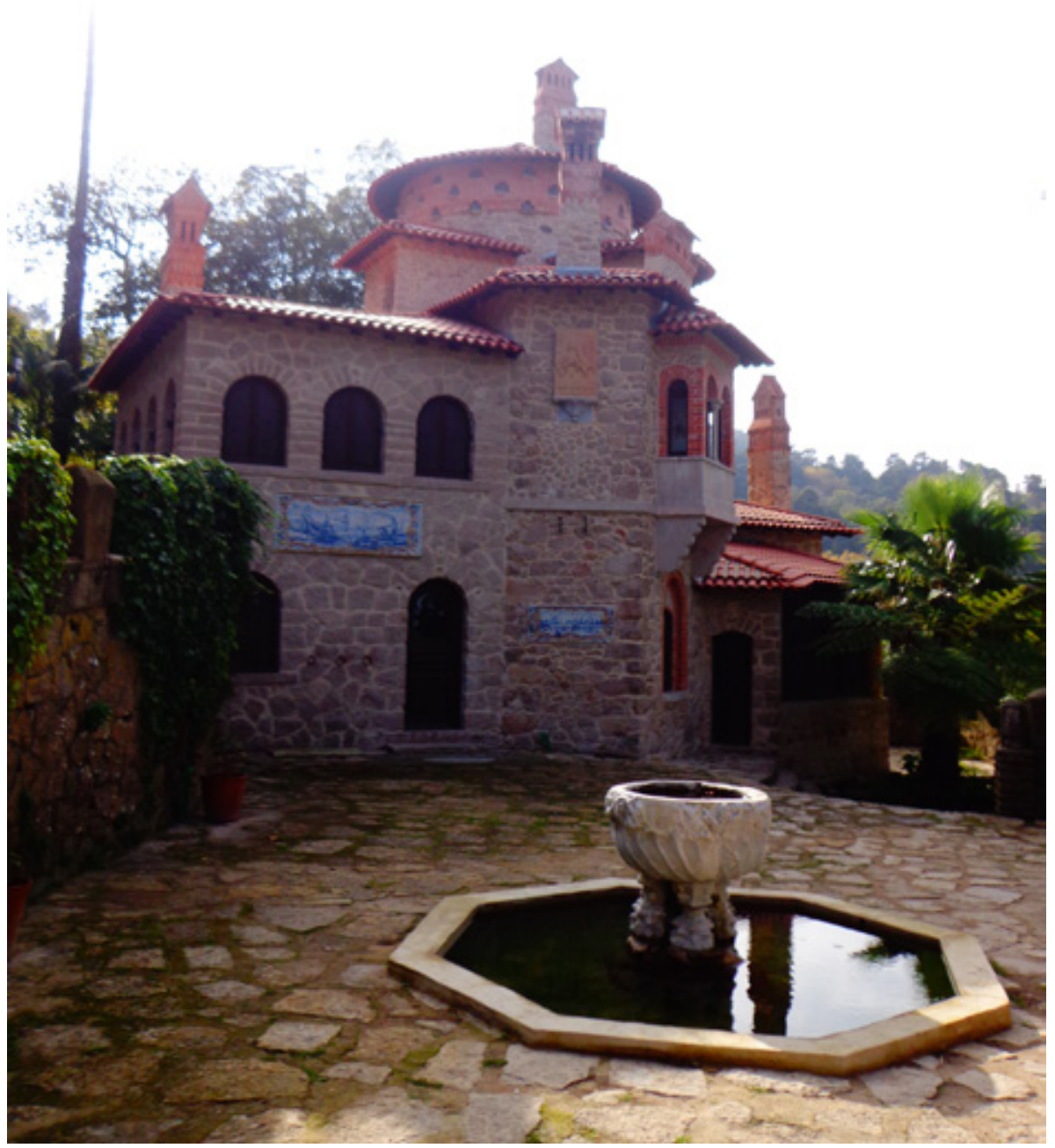

Figura 1. Luigi Manini, Villa Sassetti, 1890. Foto: Iván Moure Pazos 


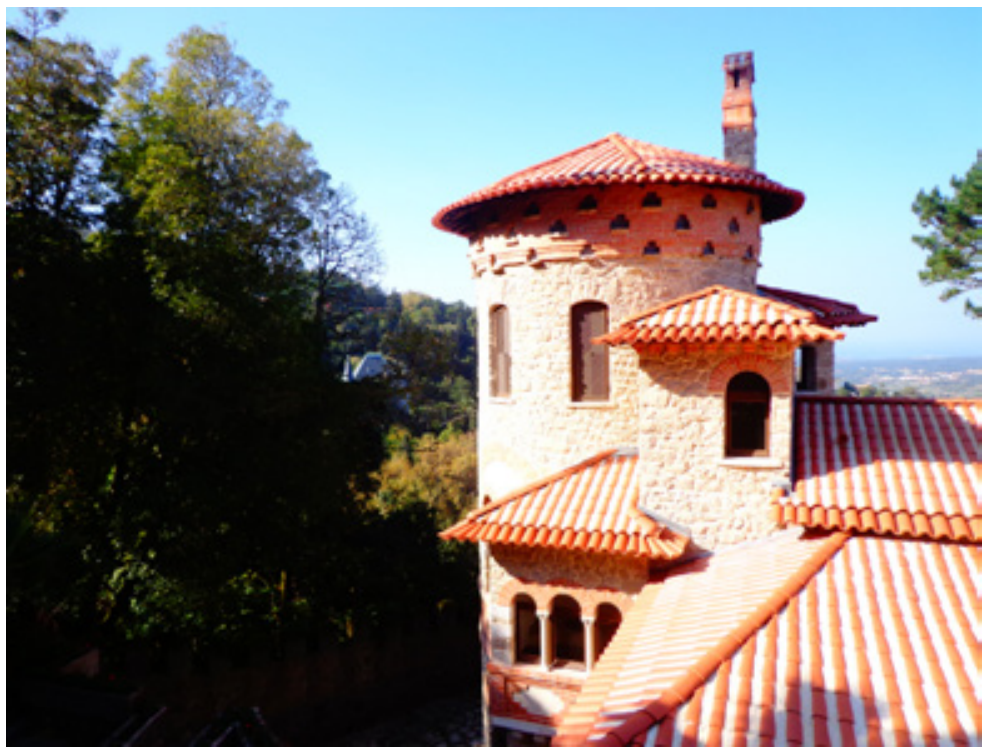

Figura 2. Luigi Manini, Villa Sassetti, 1890. Vista panorámica con el Chalet Biester al fondo. Foto: Iván Moure Pazos

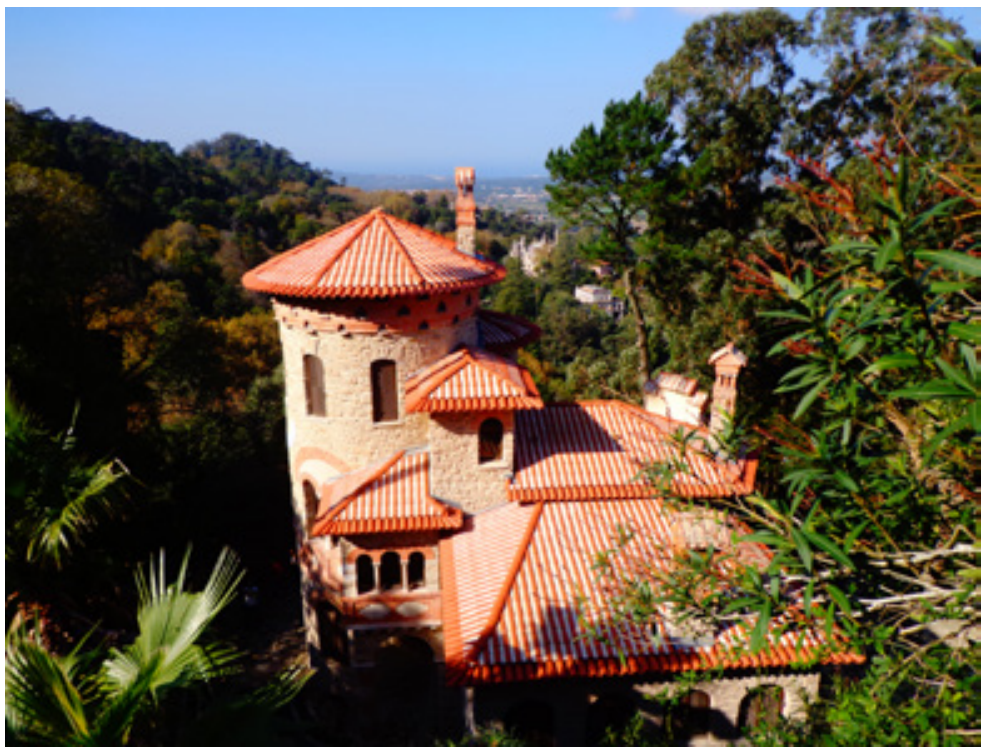

Figura 3. Luigi Manini, Villa Sassetti, 1890. Vista panorámica con la Quinta do Relógio, Vila Roma, Quinta da Regaleira y Palácio de Seteais al fondo. Foto: Iván Moure Pazos 
Figura 4. Condessa de Almedina, Vista de Vila Sassetti con figura feminina, finales del siglo XIXprincipios del $\mathrm{XX}$, óleo sobre tabla, $32,5 \times 24 \mathrm{~cm}$. Fuente: http://www.cml.pt/cml.nsf.

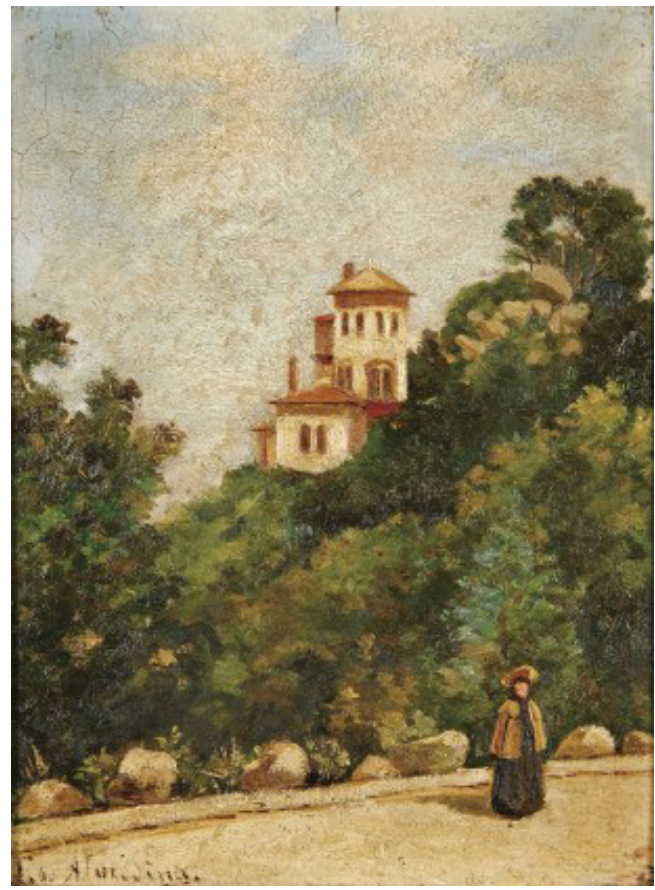

Figura 5. Luigi Manini, Alzato e piante del pianoterreno del primo progetto per la Casa Sassetti a Sintra, 1890 , lápiz y tinta roja sobre papel, 43,6 x 56,2 cm. Fuente: Museo Civico di Crema e del Cremasco, Crema (Lombardía).

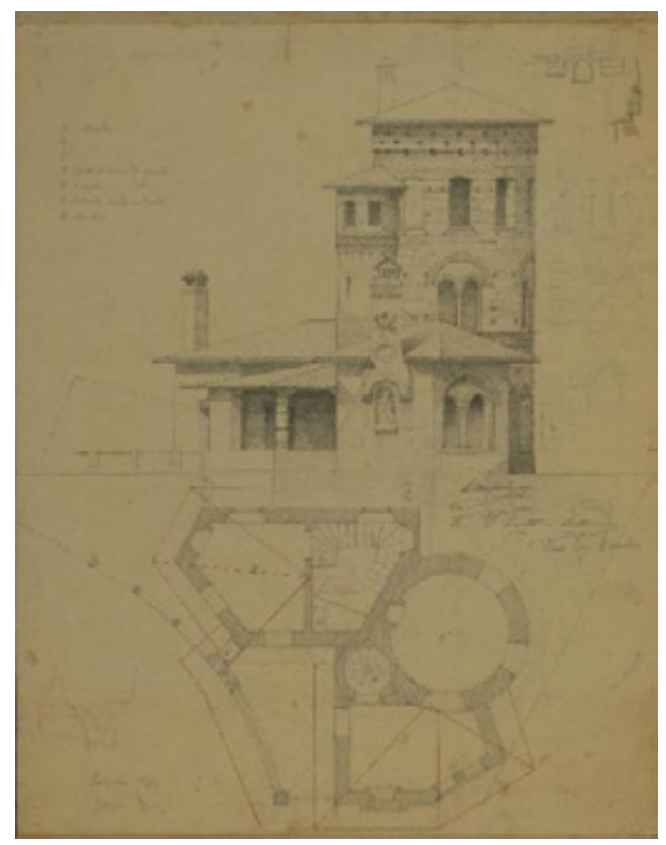
e-ISSN 2253-8305 - DOI http://dx.doi.org/10.12795/LA.2017.i29.35 


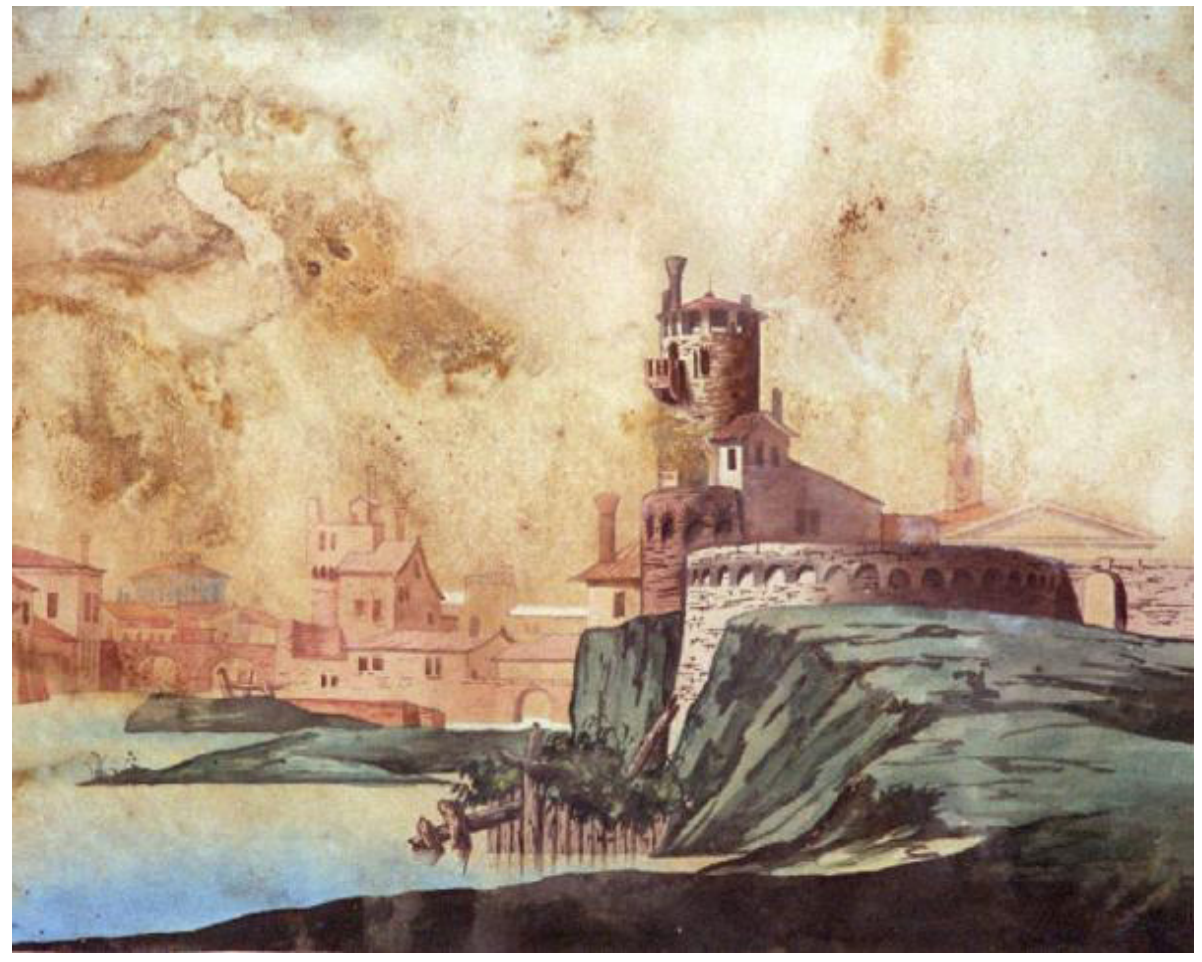

Figura 6. Luigi Manini, Veduta fantastica di città con castello o Paesaggio lacustre con architetture, hacia 1862, acuarela sobre papel, 32 x $42 \mathrm{~cm}$. Fuente: Museo Civico di Crema e del Cremasco, Crema (Lombardía). 


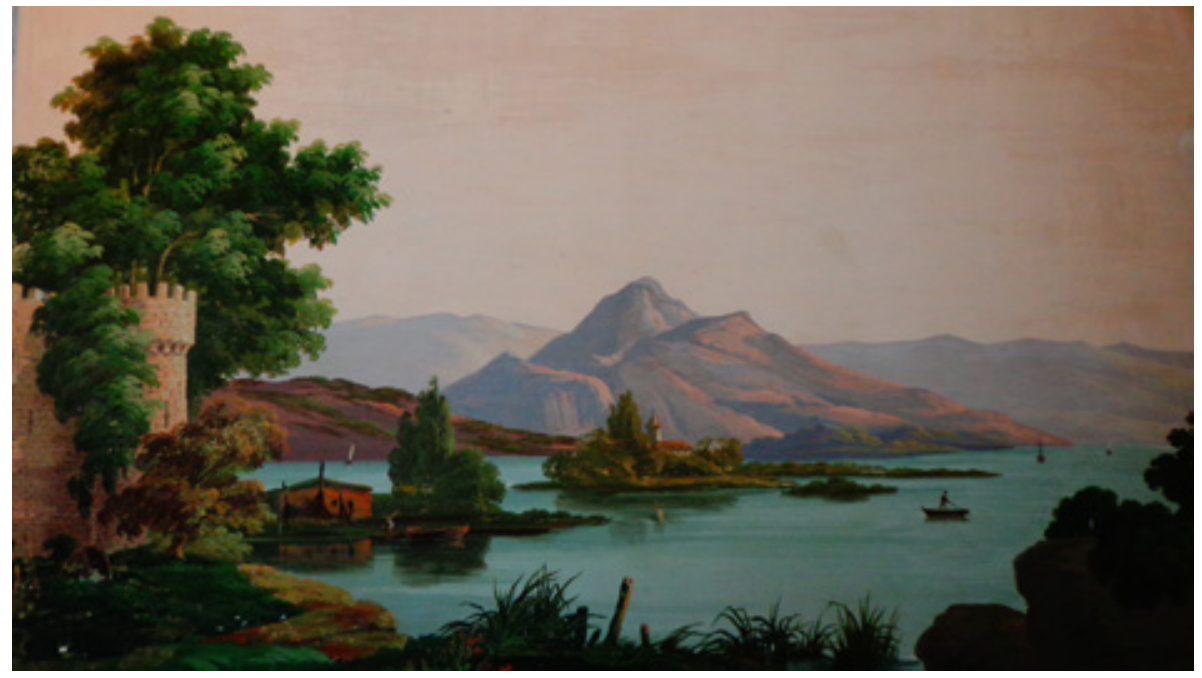

Figura 7. Atribuido a Luigi Manini, Hotel Victor, senza titolo, hacia 1885, fresco. Foto: Iván Moure Pazos

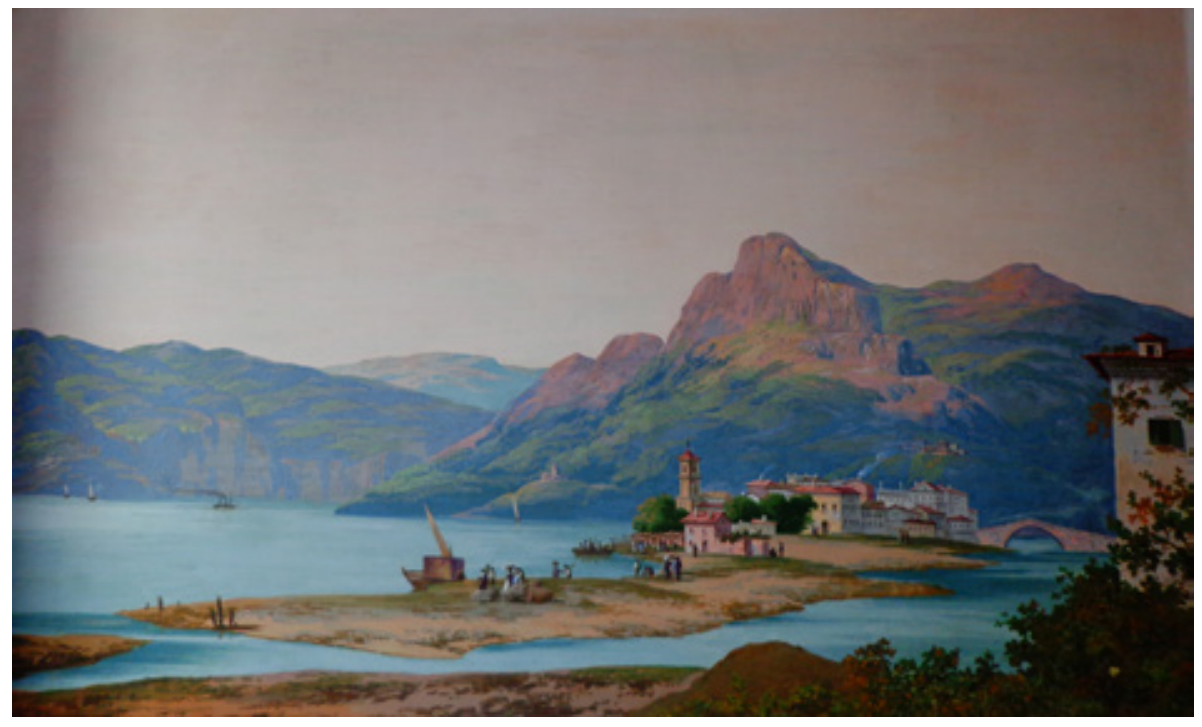

Figura 8. Atribuido a Luigi Manini, Hotel Victor, senza titolo, hacia 1885, fresco. Foto: Iván Moure Pazos 


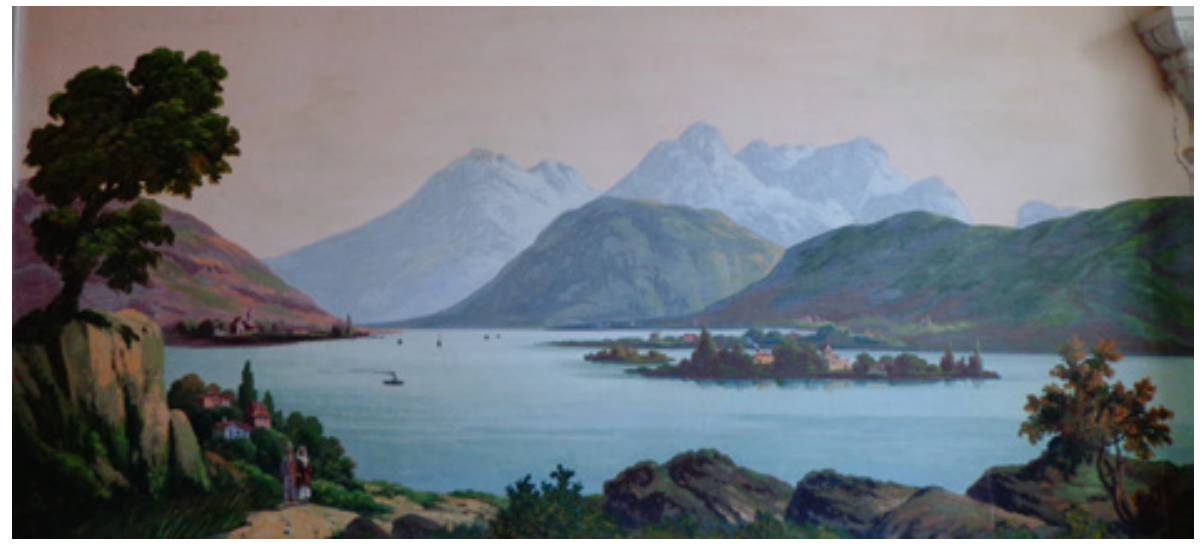

Figura 9. Atribuido a Luigi Manini, Hotel Victor, senza titolo, hacia 1885, fresco. Foto: Iván Moure Pazos

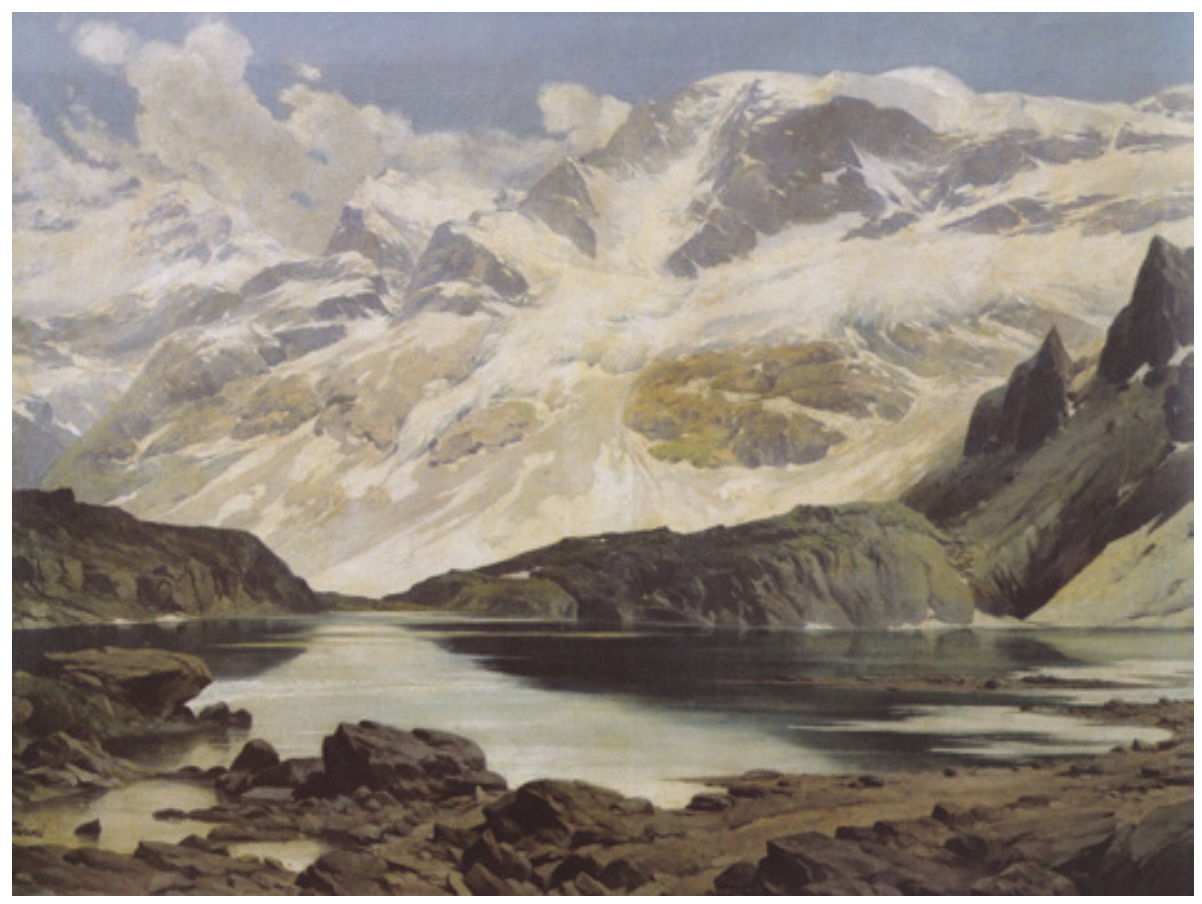

Figura 10. Luigi Manini, Paesaggio alpino con laghetto, sin fechar, óleo sobre tela, 96 x $129 \mathrm{~cm}$. Foto: Colección privada. 